participants as they doffed, and we photographed their scrubs and exposed skin before and after each donning and doffing episode. We reviewed videos for doffing errors and photographs for fluorescent spots. We counted fluorescent spots and noted their locations. Results: Overall, 45 (64.3\%) participants were women, 31 (44.3\%) were nurses, 24 (34.3\%) were physicians. Among the participants, $25(35.7 \%)$ had $>15$ years of experience and $61(87.1 \%)$ had some training in doffing. Participants frequently contaminated their skin or clothing while doffing (Table 1). For all scenarios, hands followed by the torso were contaminated most frequently. Analysis of the videos found that touching the gown front with bare hands was the most common doffing error. Fewer participants self-contaminated when using the Doffy glove without training than when using the standard exam glove. Although most participants in the glove trial indicated that they did not need to watch the Doffy glove training video again, most had difficulty doffing the Doffy glove with the beak method. Many participants stopped doffing to answer questions when they doffed the PPE ensemble during the interruption scenario. Conclusions: Self-contamination was very common with all PPE styles and during all scenarios. Distraction did not increase the risk of contamination. However, participants often stopped doffing to answer questions, which they rarely do in practice. Watching a video was inadequate training for the beak glove-doffing method. The Doffy glove, which decreased contamination compared with the standard glove in the untrained scenario, may have advantages over standard exam gloves and should be evaluated further.

Funding: None

Disclosures: None

Doi:10.1017/ice.2020.1020

\section{Presentation Type:}

Poster Presentation

Semiquantitative Staphylococcus aureus Nasal Colony Reduction in Elective Orthopedic Surgery Reduces Surgical Site Infection Lisa Saidel, Soroka University Medical Center; Riki Yosipovich, Soroka University Medical Center; Ronit Nativ, Soroka University Medical Center; Orli Sagi, Soroka University Medical Center; Orly Shimoni, Soroka University Medical Center; Abraham Borer, Soroka University Medical Center

Background: Clean surgical procedures such as hip and knee replacement and spine surgery have a low infection complication rate; however, if infections occur, there is substantial morbidity and increased cost. Staphylococcus aureus causes 30\% of surgical site infections (SSIs). S. aureus colonization increases the risk of developing SSI. Nasal povidone-iodine 5\% application reduces the

S. aureus colonization burden. Objective: We aimed to reduce SSIs by applying nasal povidone-iodine $5 \%$ prior to hip and knee replacement and spine surgery. Methods: Adult patients hospitalized for elective hip replacement, knee replacement, or spine surgeries from February 2018 through August 2019 comprised the study cohort. All patients received preoperative guidance in the outpatient clinic. On admission, the evening before surgery, a nasal swab for $S$. aureus colonization was performed, followed by povidone-iodine $5 \%$ application in both nostrils and body chlorhexidine gluconate $4 \%$ bathing. Application of these substances was repeated in the morning before surgery. Within 24 hours after surgery, an additional nasal swab for S. aureus colonization was taken. A 90-day follow-up for SSI was done. Data were compared with a similar 2016-2017 cohort without the
Table 1.

Table 1: Pre- and post-surgery data of the present cohort

\begin{tabular}{|l|l|l|l|l|}
\hline $\begin{array}{l}\text { Semi- } \\
\text { quantitative } \\
\text { nasal culture } \\
\text { results }\end{array}$ & $\begin{array}{l}\text { Spine } \\
\text { 24-h pre- } \\
\text { surgery } \\
(\mathrm{N}=101)\end{array}$ & $\begin{array}{l}\text { Spine } \\
\text { 24-h post- } \\
\text { surgery } \\
(\mathrm{N}=101)\end{array}$ & $\begin{array}{l}\text { Hip and knee } \\
\text { 24-h pre- } \\
\text { surgery } \\
(\mathrm{N}=85)\end{array}$ & $\begin{array}{l}\text { Hip and knee } \\
\text { 24-h post- } \\
\text { surgery } \\
(\mathrm{N}=85)\end{array}$ \\
\hline Negative & 84 & 94 & 65 & 74 \\
\hline $\begin{array}{l}\text { MSSA scant } \\
\text { growth }\end{array}$ & 12 & 5 & 15 & 10 \\
\hline $\begin{array}{l}\text { MSSA } \\
\text { confluent } \\
\text { growth }\end{array}$ & 3 & 0 & 4 & 0 \\
\hline $\begin{array}{l}\text { MRSA scant } \\
\text { growth }\end{array}$ & 1 & 2 & 1 & 1 \\
\hline $\begin{array}{l}\text { MRSA } \\
\text { confluent } \\
\text { growth }\end{array}$ & 1 & 0 & 0 & 0 \\
\hline
\end{tabular}

preoperative measures. Results: In total, 186 patients were included: 85 underwent hip or knee replacement (age $69 \pm 13.2$ y, $66 \% \mathrm{men}$ ), and 101 spine surgery (age $55 \pm 15.4 \mathrm{y}, 52 \% \mathrm{men}$ ). At screening, $18.3 \%$ were colonized with methicillin-sensitive $S$. aureus and $1.6 \%$ colonized with methicillin-resistant $S$. aureus. Pre- and postsurgery data for the cohort appear in Table 1 . The SSI rate per 100 patient days for hip and knee replacement and for spine surgery decreased from 0.29 and 4.0, respectively, in the 2016-2017 cohort to 0 and 0.99 , respectively, in the present cohort. Conclusions: Nasal povidone-iodine 5\% reduced the $S$. aureus colonization burden. The SSI rate in elective hip and knee replacement and spine surgery significantly decreased. We show that there is no need for $S$. aureus eradication presurgery; semiquantitative $S$. aureus nasal colony reduction should suffice in reducing SSIs. Further research is needed using quantitative measurements for $S$. aureus colonization.

Funding: None

Disclosures: None

Doi:10.1017/ice.2020.1021

\section{Presentation Type:}

Poster Presentation

Seroprevalence of Measles in Healthcare Workers in South Korea

Sun Kyung Kim, Office for Infection Control, Asan Medical Center; Jiwon Jung, Office for Infection Control, Asan Medical Center, Seoul, Korea; Sun Hee Kwak, Office for Infection Control, Asan Medical Center, Seoul, Korea; Min Jee Hong, Office for Infection Control, Asan Medical Center, Seoul, Korea; Eun Ok Kim, Office for Infection Control, Asan Medical Center, Seoul, Korea; Sung-Han Kim, Office for Infection Control, Asan Medical Center, Seoul, Korea

Background: Measles is a highly contagious disease that is transmissible by airborne particles but is preventable by vaccination. South Korea has maintained a highly immunized adult population; however, small local outbreaks of measles continued to occur, and there have been some reports of pockets of underimmunity among the young adult population. It is important to know the seroepidemiology of healthcare workers (HCWs) for policy-making process, but data on the seroprevalence of measles in HCWs in South Korea are limited. Methods: We investigated the seroprevalence of HCWs at Asan Medical Center, a 2,705-bed tertiary-care hospital in Seoul, South Korea, with 8,329 HCWs. In 2014, after an outbreak of measles occurred in a university in Seoul, Asan Medical 